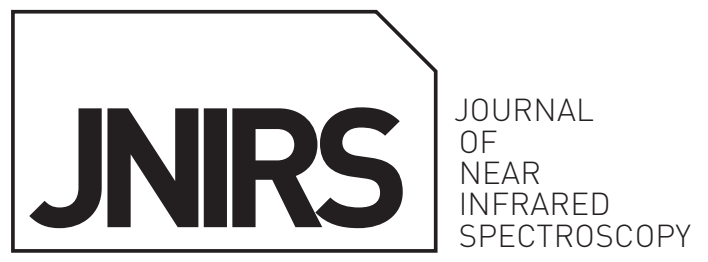

Special Issue on Medical Applications

\title{
Review \\ Brain and muscle near infrared spectroscopy/imaging techniques
}

\author{
Davide Contini, ${ }^{\mathrm{a},}{ }^{*}$ Lucia Zucchelli, ${ }^{\mathrm{a}}$ Lorenzo Spinelli, ${ }^{\mathrm{b}}$ Matteo Caffini, ${ }^{\mathrm{c}}$ Rebecca Re, ${ }^{\mathrm{a}}$ Antonio Pifferi, ${ }^{a}$ \\ Rinaldo Cubeddu ${ }^{\mathrm{a}}$ and Alessandro Torricellia \\ aPoltecnico di Milano, Dipartimento di Fisica, Piazza L. da Vinci, 32 Milan, Italy. E-mail: davide.contini@polimi.it \\ ${ }^{b}$ Istituto di Fotonica e Nanotecnologie, IFN-CNR, Piazza L. da Vinci, 32 Milan, Italy \\ 'Department of Health Sciences, University of L'Aquila, L'Aquila, Italy
}

\begin{abstract}
This review is an introduction to functional near infrared spectroscopy techniques and instrumentation which are commonly used for the estimation of haemoglobin concentration in muscle and brain cortex. Three approaches are considered: continuous wave, frequency domain and time domain near infrared spectroscopy. The different techniques are briefly analysed from a theoretical point of view in order to understand their capabilities and drawbacks. Some of the instrumentation commonly used is considered, presenting the main characteristics of the different techniques, their similarities and principal differences.
\end{abstract}

Keywords: functional near infrared spectroscopy, continuous wave NIR instruments, frequency domain, time domain, photon migration

\section{Introduction}

White paint, foam, fog, clouds and biological tissues have a common feature: all of them appear visually opaque. This particularity is due to the fact that visible light travelling in these materials experiences scattering phenomena rather than absorption. Inside these materials (diffusive media), photons can be roughly described as bouncing balls in a random structure: photons travel in random pathways caused by thousands of elastic scattering events; only a few of these photons which encounter a chromophore (absorbing centre) will be lost.

Multiple scattering introduces an uncertainty in the path length travelled by photons; therefore, light propagation in diffusive media is termed photon migration. Accordingly, the key parameters of light propagation in diffusive media are the scattering length $\left(l_{s}\right)$ and the absorption length $\left(l_{a}\right)$, which represent, respectively, the mean path between two conse- quent scattering and absorption events. The inverse of $l_{\mathrm{s}}$ and $l_{\mathrm{a}}$ are, respectively, named scattering coefficient $\left(\mu_{\mathrm{s}}\right)$ and absorption coefficient $\left(\mu_{\mathrm{a}}\right)$, representing the scattering and absorption probability per unit length. Normally, the diffusive properties of these materials are described in terms of the reduced scattering coefficient $\left(\mu_{\mathrm{s}}{ }^{\prime}\right)$, which can be expressed as $\mu_{s}{ }^{\prime}=(1-g) \mu_{s}$, where $g$ is the anisotropy factor (i.e. the average cosine of the angle between the photon directions before and after the scattering event). The reduced scattering coefficient is used as a comprehensive parameter because it takes into account not only the probability of a scattering event but also the average scattering direction. In particular, for biological tissues in the visible and near infrared (NIR) range, $g$ is close to 0.9 , indicating that photons are scattered predominantly in the forward direction and this implies that many scattering events are necessary in order to consider the fully randomised 
initial propagation direction of a photon. In fact, the reduced scattering coefficient can be interpreted as the reciprocal of the average distance that a photon travels in diffusive media before its initial direction could be considered completely randomised.

\section{Near infrared spectroscopy}

The aim of diffuse light imaging and spectroscopy is to monitor tissue physiology in depth la few centimetres below the tissue surface), ${ }^{1}$ Here, we cannot consider traditional optical spectroscopy or traditional microscopy because traditional techniques require optically thin samples. In addition, absorption cannot be high because light should be able to reach tissue structure located centimetres below the surface. In the visible-NIR range, the absorption of the main constituents of biological tissues (haemoglobin, water, lipid and collagen) is low ( $\mu_{a}$ lower than $0.5 \mathrm{~cm}^{-1}$ ), while the scattering due to organelles and other microscopical refractive index mismatch is large $\left(\mu_{\mathrm{s}}{ }^{\prime}\right.$ in the range between $5 \mathrm{~cm}^{-1}$ and $25 \mathrm{~cm}^{-1}$ ). The photon migration in biological tissue can be described with a diffusion equation because scattering is predominant with respect to absorption (for further details see Martelli et al. ${ }^{2,3}$ ). Considering the photon fluence rate at a certain wavelength, $\phi(r, t)$, Inumber of photons per unit area and time: photons $\mathrm{cm}^{-2} \mathrm{~s}^{-1}$ ), it is possible to derive from an energy balance in a small volume inside the medium:

$$
\frac{\partial \Phi(\mathbf{r}, t)}{\partial t}=\nabla \cdot D(\mathbf{r}) \nabla \Phi(\mathbf{r}, t)+\nu S(\mathbf{r}, t)-\nu \mu_{\mathrm{a}}(\mathbf{r}) \Phi(\mathbf{r}, t)
$$

where $D$ is the diffusion coefficient, $D(\mathbf{r}) \cong v / 3 \mu_{\mathrm{s}}{ }^{\prime}(\mathbf{r})$, and $\nu$ is the speed of light inside the medium. The left hand side of Equation (1) is the increasing rate of the number of photons inside the considered volume that is equal to the balance between the number of photons scattered from other directions inside the volume per unit time, the photons generated by light sources eventually present inside the volume [respectively first and second terms in the right hand side of Equation (1)] and the number of photons which are absorbed inside the volume per unit time [third term in the right hand side of Equation (1)]. Another important feature is that, in the NIR range, the spectra of major tissue chromophores differ significantly (see Figure 1). In particular, oxygenated haemoglobin $\left(\mathrm{HbO}_{2}\right)$ and deoxygenated haemoglobin $(\mathrm{HHb})$ can be discriminated on the basis of their spectral fingerprint ${ }^{4}$ and, therefore, diffuse optical methods are sensitive to haemodynamic and tissue oxygenation. In fact, by quantifying both $\mathrm{HbO}_{2}$ and $\mathrm{HHb}$, it is possible to estimate the total content of haemoglobin (sum of the two haemoglobin species), the oxygen saturation (ratio between oxy- and total haemoglobin) and to follow their evolution with repetitive measurements. These features make NIR spectroscopy particularly powerful for the investigation of a variety of problems. For example, the greater vascularisation and oxygenation of tumours in comparison with the surrounding tissues can be used in order to detect malignant tissue by means of optical techniques. A similar approach can be applied to identify brain bleeding or changes in brain cortex oxygenation associated with brain activation, or muscle physiology and function.

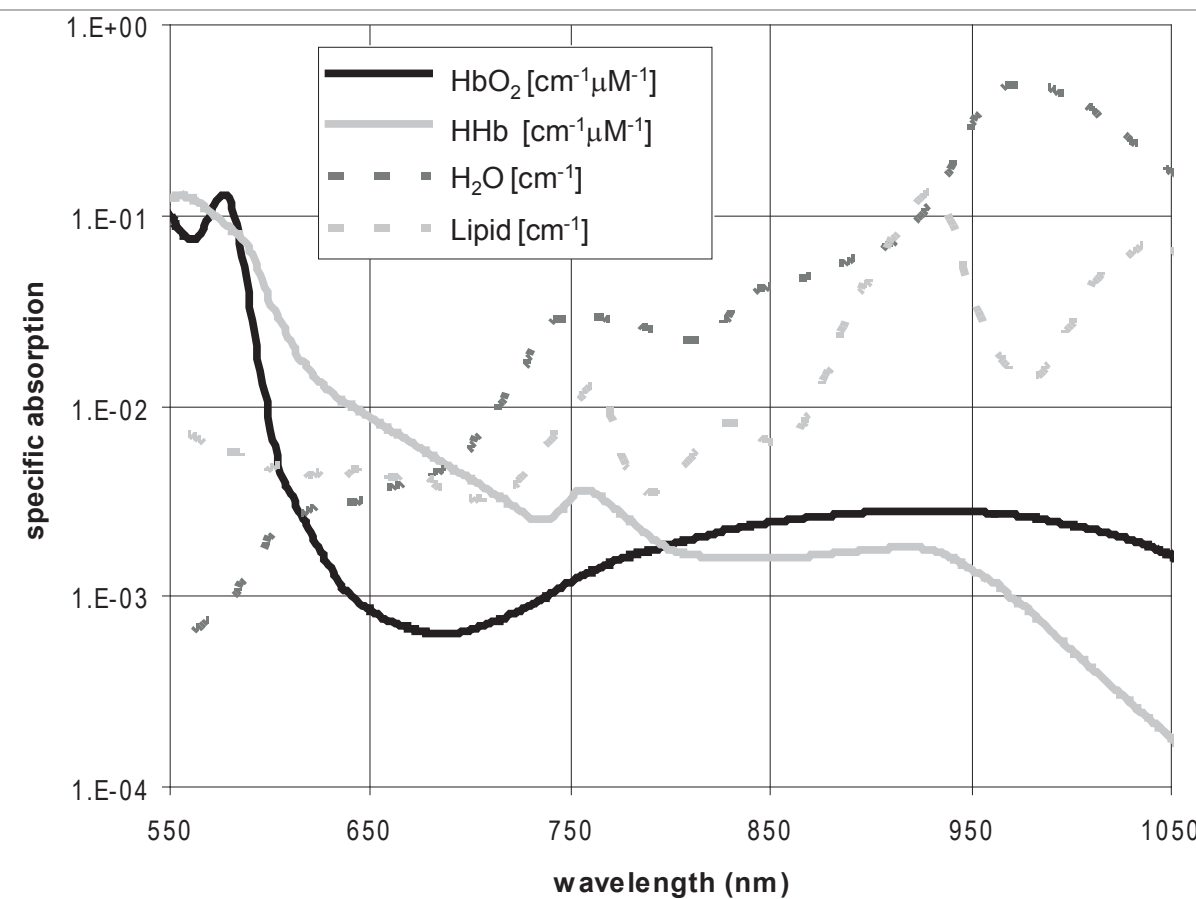

Figure 1. Absorption spectra of main human body tissue constituents $\left(\mathrm{HbO}_{2}, \mathrm{HHb}\right.$, lipid and water) in the range between $550 \mathrm{~nm}$ and $1050 \mathrm{~nm}$. 
The absorption coefficient at a certain wavelength can be expressed as a function of the chromophore concentration inside the medium:

$$
\mu_{a}(\lambda)=\varepsilon(\lambda) c
$$

where $\varepsilon(\lambda)$ is the molar specific absorption coefficient array $\left(\mathrm{M}^{-1} \mathrm{~cm}^{2}\right)$ and $\mathrm{c}\left(\mathrm{M} \mathrm{cm}^{-3}\right)$ is the molar concentrations array. To evaluate the concentrations of $n$ chromophores of interest, it is necessary to measure the absorption coefficient for $n$ different wavelengths in order to solve the linear system composed by $n$ equations, each similar to Equation (2) lone for each wavelength). In particular, if we are interested in the tissue oxygenation, we need to evaluate $\mu_{\mathrm{a}}$ at two different wavelengths in order to discriminate $\mathrm{HbO}_{2}$ and $\mathrm{HHb}$ (regarding the wavelength selection, see the Instrumentations section).

There are three different approaches to measure this optical parameter. They differentiate in terms of the type of light sources employed: continuous wave (CW) light, amplitude-modulated light or frequency domain (FD) approach and pulsed light or time domain (TD) approach (see Figure 2). Obviously, the choice of the light source influences the instrumental apparatus, its cost and the models applied to analyse data. In the following sections, the different techniques are presented from a theoretical point of view while, in the last section of the paper, we focus our attention on different instrumental approaches.

\section{Continuous wave technique}

This technique measures the attenuation of light injected into a sample. In a transparent (non-diffusive) material this simple measurement should be sufficient to evaluate the absorption coefficient of the medium: from the Beer-Lambert law we can calculate the absorption coefficient, knowing the distance between optodes, i.e. the straight photon pathlength:

$$
A(t, \lambda)=-\ln \left[\frac{I_{\text {out }}(t, \lambda)}{I_{\text {in }}(t, \lambda)}\right]=\mu_{\mathrm{a}}(t, \lambda) d
$$

where $A(t, \lambda)$ is known as the attenuation at the time, $t$, for the wavelength $\lambda, I_{\text {out }}(t, \lambda)$ is the re-emitted light from the sample at the time, $t$, for the wavelength $\lambda, I_{\text {in }}(t, \lambda)$ is the injected light into the sample at time, $t$, for the wavelength, $\lambda$, and $d$ is the distance between optodes which, in a transparent medium, equals the pathlength of photons in the sample.

In biological tissues in the NIR range, Equation (3) cannot describe light absorption, since the signal attenuation is caused predominantly by photon scattering. Thus, the measurement of only light attenuation cannot discriminate between absorption and scattering effects. This problem can be solved by modifying Equation (3), taking into account the ulterior losses and the uncertainty regarding the photon mean pathlength which is introduced by scattering. Adding these correction factors and expanding $\mu_{a}$, Equation (3) becomes:

$$
A(t, \lambda)=c(t) \cdot \varepsilon(\lambda) \cdot \rho \cdot \operatorname{DPF}(\lambda)+G(\lambda)
$$

where $\operatorname{DPF}(\lambda)$ is the differential pathlength factor accounting for the increase in pathlength $(\rho)$ between the source and detector for light being scattered and $G(\lambda)$ is the term accounting for the loss of photons due to scattering. Equation (4) takes the name of modified Beer-Lambert Law. 5,6 With this equation, an absolute estimation of chromophore concentration is impossible without a measurement of $G(\lambda)$. However, changes in the chromophore concentrations can be evaluated considering that the scattering coefficient does not vary:

$$
\partial c=\frac{\partial A(\lambda)}{\varepsilon(\lambda) \cdot \operatorname{DPF}(\lambda)}
$$

The accuracy of Equation (5) is affected by the scarce knowledge of $\operatorname{DPF}(\lambda)$ that should be measured. Normally values for the DPF are tabulated but the heterogeneity of population and tissues types are not considered; for this reason, it is important to clearly state which DPFs were used. However, this approach is interesting and useful for many applications, thanks to the excellent signal-to-noise ratio and to the manageable configuration that $\mathrm{CW}$ systems normally exhibit. Measurements of the attenuation of light between two points on the surface of a tissue are not only straightforward and inexpensive, but also contain a remarkable amount of useful information, as demonstrated by clinical successes.?

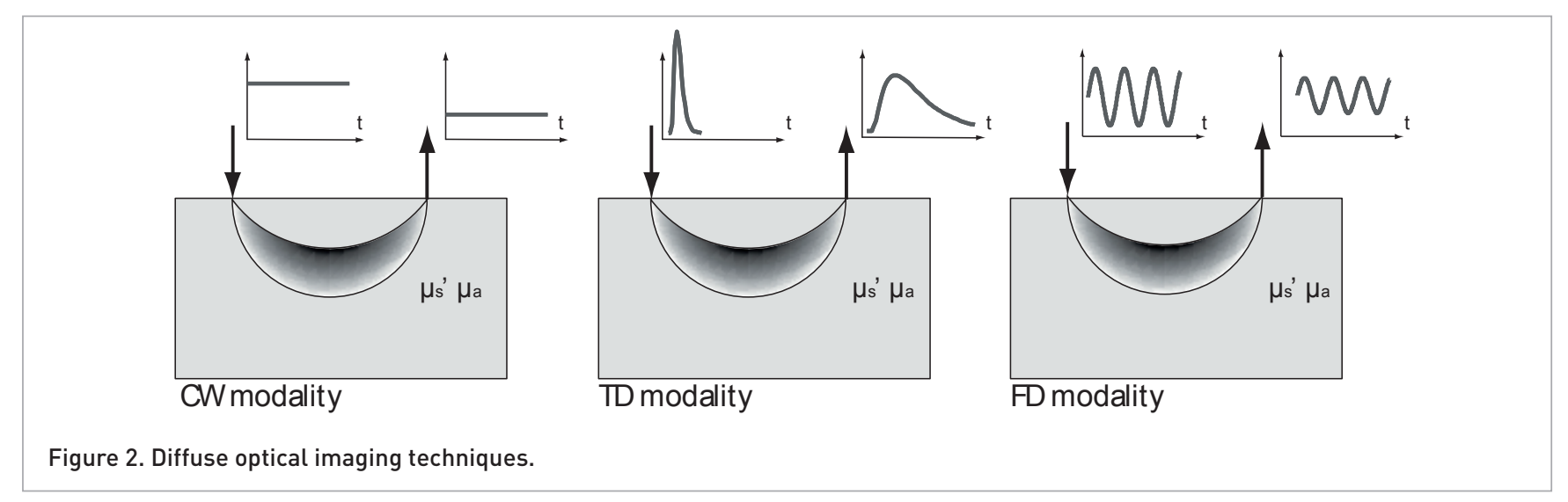


Patterson et al. ${ }^{8}$ proposed a methodology for the absolute assessment of the tissue absorption coefficient exploiting CW measurements performed at different inter-optodes distances (in the range from $3 \mathrm{~cm}$ to $4.5 \mathrm{~cm}$ ). They demonstrated that in the diffusion approximation:

$$
\mu_{\mathrm{a}} \mu_{\mathrm{s}}^{\prime} \cong \frac{1}{3}\left(\frac{\partial A}{\partial \rho}-\frac{2}{\rho}\right)^{2}
$$

where $\rho$ is the inter-optodes distance. Measuring the linear interpolation of light attenuation at different inter-optodes distances and estimating the reduced scattering coefficient, it is possible to calculate the absorption coefficient without using the DPF. The accuracy of the results depends on the knowledge of $\mu_{s}{ }^{\prime}$, but simply considering the $\mu_{s}{ }^{\prime}$ dependence on wavelength (Mie scattering theoryl and performing measurement of $A(t, \lambda)$ at different wavelengths it is possible to estimate oxygen saturation. This is a significant advantage; in fact, only using the DPF methodology, changes in $\mathrm{HbO}_{2}$ and $\mathrm{HHb}$ concentrations can be retrieved. The multi-distance approach is often used in CW instrumentation lone of the first examples was reported by Matcher et al. ${ }^{9}$ ).

There are certain disadvantages associated with $\mathrm{CW}$ imaging. ${ }^{10}$ The first is that intensity measurements are more sensitive to the optical properties of the superficial layer of tissues rather than to the optical properties of regions localised deeper within the tissue. This is due to the characteristic "banana" shape (see Figure 3) of the volume of tissue over which the measurement is sensitive (known as the photon measurement density function), which is narrow near sources and detectors and very broad in regions between them. ${ }^{11,12}$ For the same reason, the detected intensity is highly dependent on surface coupling. In fact, the presence of hair or local variation in skin colour can also have a major influence on intensity measurements. ${ }^{1}$ The increase of separation between light injection and collection enhances the depth sensitivity of CW measurement, because the photon measurement density function is enlarged and its extension reach deeper regions inside the medium (normally the inter-optodes distance is around $3-4 \mathrm{~cm}$ ). However, the sensitivity to superficial layers is always an issue and systemic changes in the optical properties of tissue can be confused or misinterpreted as changes in depth. This aspect can be partially resolved by performing measurements at two different inter-optode distances: a small one (less than $1 \mathrm{~cm}$ ) and a larger one $(3-4 \mathrm{~cm}$ ); in this way, the small distance optodes will investigate only the superficial layer of the tissue probing the systemic changes in haemoglobin concentration, while the larger distance optodes will probe both the superficial and deeper tissue regions. Information obtained from the former measurement can be used to correct information of the latter one, decreasing its dependence on superficial/systemic optical parameter changes and increasing the signal contrast due to the deeper ones.

\section{Time domain technique}

Time domain (TD) systems employ pulsed laser sources. The temporal distribution of re-emitted photons when a pulse of light propagates through a highly scattering medium is generally known as the temporal point spread function (TPSF), which normally extends over several nanoseconds. We can consider the TPSF, in which the information about tissue optical properties is encoded, as the impulse response of the tissue. In contrast to CW measurements, TD measurements take into account the effect of multiple scattering, since photon pathlength is directly related to the time-of-flight in the medium. Following the injection of the light pulse in a turbid medium, the temporal distribution of the re-emitted photons will be delayed, broadened and attenuated. To a first approximation, the delay is a consequence of the finite average time that photons take to cover the distance between source and detector. Broadening is mainly due to the many different paths that photons undergo as a result of multiple scattering. Finally, attenuation is observed since absorption reduces the probability of detecting a photon and diffusion into other directions within the medium decreases the number of detected photons in the considered direction. In particular, absorption affects the asymptotic slope of the TPSF tail. ${ }^{14}$ Thus, in principle, it is possible to discriminate absorption and scattering coefficients

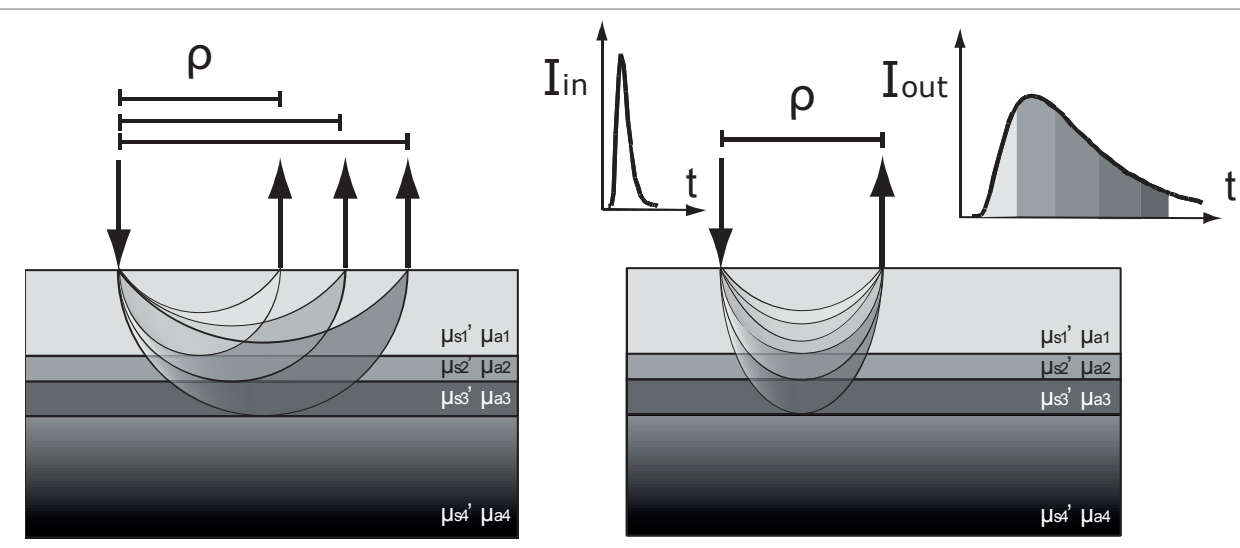

Figure 3. Dependence of the depth sensitivity on inter-fibre distance for continuous wave (CW) measurements (left side) and dependence of the depth sensitivity on arrival time for time domain (TD) measurements (right side). 
independently, for instance by fitting the experimental TPSF with a suitable theoretical model describing the propagation of photons in a highly diffusive medium. ${ }^{15}$ However, the most important feature of the time-resolved approach is that the penetration depth is naturally encoded in the photon arrival time distribution. In fact, photons that travel for a few hundreds of picoseconds inside the medium have a low probability of reaching the deeper region of the tissue, while photons that spend more time (nanoseconds) within the tissue have a greater probability of encoding signals from tissue in depth.

By solving Equation (1), it is possible to obtain the photon time distribution exiting a turbid medium at a certain distance from the light injection point (for a detailed description see Martelli et al. ${ }^{2}$ ). For a simple geometry, where injection and detection point are on the same sample surface (typical arrangement for brain or muscle measurements) and for the simplest case of a semi-infinite medium in which a delta like laser pulse is injected, the solution of Equation (1) is:

$$
R(\rho, t)=\frac{1}{2(4 \pi D \nu)^{3 / 2} t^{5 / 2}} \exp \left(-\frac{\rho^{2}}{4 D \nu t}\right) \exp \left(-\mu_{\mathrm{a}} \nu t\right) B\left(\mu_{\mathrm{s}}{ }^{\prime}, t\right)
$$

where $R(\rho, t)$ is the TPSF evaluated at a distance $\rho$, while $B\left(\mu_{\mathrm{s}}{ }^{\prime} t\right)$ is a term depending on the reduced scattering coefficient and the boundary conditions. ' Looking at Equation (7), it is worth noting that $\mu_{\mathrm{a}}$ and $\mu_{\mathrm{s}}{ }^{\prime}$ are completely decoupled, thus it is possible to estimate the absolute value of absorption coefficient by fitting the experimental data with the proper theoretical model and calculating the absolute values of chromophore concentrations. Knowledge of the temporal distribution of photon overcomes the first problem of CW measurements: the impossibility to directly obtain absolute quantifications. Obviously, the accuracy of the fitting method depends on the accuracy of the theoretical model and on the knowledge of the bulk optical properties. ${ }^{16}$ Analytical solutions of Equation (1) can be obtained only in simple cases ${ }^{2}$ (planar geometry and simple structures), for more complex geometry and sample structures (for example the head), numerical solutions based on the finite element method approach ${ }^{12}$ or based on Monte Carlo simulations ${ }^{17}$ can be employed. The fitting procedure shows a lower signal-to-noise ratio than the analysis of CW measurements with the modified Beer-Lambert law. Often, during functional measurements, in particular of brain activity, changes of $\mathrm{HbO}_{2}$ and $\mathrm{HHb}$ concentrations have very low contrast (a few percent).

The second, and main, feature of TD measurements is that depth information is naturally encoded in time and is independent of the distance between injection and detection points, in contrast to the CW measurements (see Figure 3). In fact, by analysing the first part of TPSF, information coming from the superficial layer is enhanced, while, focusing the attention on the tail of TPSF, information coming from deeper layers is obtained. It would be possible to exploit this feature in order to discriminate between systemic haemodynamic changes occurring in the first layers of tissue (for example skin) and deeper ones related with brain or muscle activity. ${ }^{18-20}$ There are mainly two approaches to exploit this feature: the first is the time-gated approach, while the second is the moments approach. Time gating involves dividing the TPSF in slices centred at different times. The changes in the number of photons in each slice between two different TPSF lone used as reference status and the second measured during an activated status), permit the calculation of the changes in the absorption coefficient caused by the functional activation of the tissue. In particular, the first slices of the TPSF give information about haemodynamic variations which have occurred in superficial layers, while the last slices of the TPSF give information about deeper haemodynamic variations. ${ }^{18}$ The moments approach exploits the different depth sensitivity shown by the TPSF moments. Integral and the first moment of TPSF are mainly sensitive to superficial changes (the integral of TPSF is equivalent to the CW measurement), while the second moment of TPSF (the variance) is sensitive to deeper changes. ${ }^{19,20}$ In contrast to time gating, moments are independent on instrumental characteristics; however, the calculation of chromophore concentration calculations starting from variance is not straightforward.

It can be beneficial to apply both types of data analysis in order to exploit both features of TD measurements: the possibility to obtain absolute measurement and to determine the signal provenance. In fact, the haemodynamic basal level can be estimated by applying the FIT procedure to TPSFs acquired during the resting state, overcoming the low signalto-noise ratio of this procedure simply by averaging TPSFs acquired over a period of seconds. Focal changes in optical properties due to functional activity of the brain cortex or muscle can be estimated by applying the time gating or moment procedure.

\section{Frequency domain technique}

In principle, time-resolved data can be translated in the frequency domain (FD) by exploiting the Fourier transform. This implies that the information contained in data should be the same, and it is possible to discriminate absorption and scattering coefficients and to obtain absolute quantification of chromophore concentrations. The idea on which FD is based is to directly acquire frequency data employing sources that are amplitude modulated and to measure the reduction in amplitude and phase shift of the output signal. Absorption and scattering affect amplitude and phase differently: amplitude encodes information regarding $\mu_{a}$ and phase measurements are used in order to evaluate the photon pathlength instead of estimating it as in CW measurements. It is also possible to write the diffusion equation in the frequency domain and to solve it as in the time domain, ${ }^{21}$ fitting $\mu_{a}$ and $\mu_{s}{ }^{\prime}$ directly. The choice of the modulation frequency establishes the achievable spatial and temporal resolution and the sensitivity to absorption and scattering coefficients.

TPSF inevitably contains more information than a single phase and amplitude measurement at one source frequency; in fact, the frequency content of the TPSF extends up to several gigahertz. FD measurements should be performed at several 
modulation frequencies in order to collect the same information as a TD measurement.

\section{Instrumentation}

Instrumentation for functional NIR spectroscopy of muscle or brain is based on four main components: sources, systems for injection/collection of the light, detectors and acquisition/ processing of the signal. The particular characteristics of each component depend on the type of measurement scheme implemented: CW, TD or FD. However, some components are independent of the type of instrumentation.

The choice of the light source wavelengths does not depend on its modulation but only on the spectral fingerprints of the chromophores of interest and on the background optical properties of the surrounding tissue. In particular, for the study of the oxygenation in muscle and brain activity, the two wavelengths in the NIR range used for the discrimination of $\mathrm{HbO}_{2}$ and $\mathrm{HHb}$ are normally chosen on the opposite sides of their isosbestic point (around $800 \mathrm{~nm}$ ), in order to maximise the accuracy in the discrimination of the two haemoglobin species. ${ }^{10}$ Obviously, with this choice, the total absorption of the entire tissue must be taken into account in order to have a sufficient signal-to-noise ratio to explore tissues in depth. In this framework one wavelength should be in the range between $650 \mathrm{~nm}$ and $760 \mathrm{~nm}$ and the other around $830 \mathrm{~nm}^{22-24}$ (see Figure 1).

Light injection into the tissue and the collection of the remitted photons show the same issues for the three kinds of instruments (CW, TD and FD). In all three cases, light must be delivered to the tissue in one or more locations at the same time, or sequentially, and the diffused photons exiting the tissue must be collected in one or more points in order to be detected. Normally, the light is injected and collected by means of single fibre optics or a bundle of fibre optics. Only in the case of CW instrumentation it is common to couple sources and detectors with the sample, as a result of their possible miniaturisation. This layout is associated with an increase in the coupling efficiency but it needs electrical insulation in order to electrically uncouple the sample and the instruments for safety reasons. The use of optical fibres naturally solves this problem by placing the instrument in a lower risk class due to the impossibility of having a short circuit between the subject and the set-up, even if probes based on fibre optics are slightly less robust and often less flexible, increasing the discomfort of the subject. For functional measurements of brain cortex activity, the direct contact scheme can also be used in the parts of the head with hair, although the mounting of the probe can be tedious and time consuming. In some cases, the excessive time for the subject preparation can limit the use of this approach to measurements on the forehead. Whatever the coupling method used, the optical contact between the sample and the instrument is critical for obtaining good results. In fact, movement artefacts can be reduced with a proper design of the optical probe. This aspect is fundamental both for brain and muscle studies, especially during muscle contractions.
The choice of detectors for NIR spectroscopy instruments strictly depends on the type of laser sources implemented. With pulsed sources, the detectors must have a fast response and be very sensitive: up to the single photon detection, with CW sources we can use both solid state detectors and photo tube detectors operating in a simpler linear regime.

The acquisition component consists of readout and elaboration electronics for the electrical signal provided by the detector. In CW measurements, it is sufficient to read and digitalise the current proportional to the detected signal, while in frequency and time domains, the electronics are more complex. In particular, in FD, a heterodyne demodulation set-up is used to acquire phase and intensity information, while in TD a time correlated single photon counting (TCSPC) electronic chain is often employed to reconstruct the time distribution of the photons exiting the sample.

In the following sections, we give a brief overview of the most frequently used instruments in the field of NIR spectroscopy for muscle and brain studies analysing their main characteristics (see Ferrari and Quaresima ${ }^{25}$ and Cutini et al. ${ }^{26}$ for a review of these two applications).

\section{Continuous wave instruments}

Many CW instruments are already on the market for the study of brain and muscle oxygenation, ${ }^{27-37}$ as demonstrated in recent reviews. ${ }^{38-44}$

In CW regime, the sources used are light emitting diodes, ${ }^{27-31}$ which are very compact and integrable directly into the measurement probe, or laser heads ${ }^{32-37}$ which are more complex to use and to integrate, but with higher available power. We can find instruments working with two wavelengths, ${ }^{27-30,32,34}$ with three ${ }^{33,35}$ or four. ${ }^{31,37}$ Normally, in CW instruments, all the wavelengths are coupled in the tissue at the same time and in the same place and the sources are modulated at a few kilohertz in order to perform a demodulation of the detected signals to distinguish between the different wavelengths. The solution of modulating the light sources can be extended; in fact, this approach called "frequency multiplexing" can be used not only to distinguish the wavelength of the detected photon but also the spatial provenance. By multiplying the number of sources and modulating them with a slightly different frequency, it is possible to continuously shine light on the sample and simultaneously collect re-emitted photons in different positions, therefore measuring light attenuation in tens of points at the same time. The frequency multiplexing scheme has the disadvantage that the shot noise, which is not frequency specific, is combined from all light sources which are on at the same time and it cannot be separated for each light source. Another drawback of this approach is the fact that, by increasing the number of injection points and consequently the number of frequencies, the lock-in detection scheme becomes more complex, because of the increase in the number of possible demodulation frequencies. Different instruments with imaging capabilities are based on this approach; see, for example, the FOIRE $3000^{33}$ provided by Shimadzu and the ETG $-4000^{34}$ provided by Hitachi, both with 
up to 52 measurement points. A second way to obtain imaging capabilities without increasing the complexity of the detection system is based on the time multiplexing of the sources. Light is simply shone in the different injection points sequentially, by means, for example, of an optical switch. In this way, it is possible to reconstruct the entire image after each switching sequence (see, for example, NIRScout ${ }^{29}$ ). The main drawbacks of this approach are the low number of measurement points and the fact that the maximum acquisition rate is limited by the number of injection points in the sequence and by the achievable signal-to-noise ratio of a single measurement. Obviously, this second approach permits a reduction in the dimensions of the system and its costs.

There are two different modes of delivering light to the sample and collecting re-emitted photons: either by means of optical fibres or by directly integrating sources and/or detectors in the optical probe. The former is used in all cases in which the electronics for modulating the sources and/or demodulating the detected signal are bulky or when the number of injection and detection points is large..$^{29,33,34}$ The latter is used in all cases in which the number of points is limited. ${ }^{27,30-32,35,37}$ In the cases in which injection and detection systems are completely integrated in the probe, it is possible to construct a wireless system by adding a radio transmitter/receiver in the probe. ${ }^{27,30}$ The wearability of these systems limits the number of measurement points to one or two, which is not enough to realise functional brain imaging but sufficient to perform, for example, monitoring of muscle fatigue. When detectors are directly in contact with the sample, ${ }^{27,30-32,35,37}$ it is easier to implement a multi-distance measurement performing spatially resolved spectroscopy (see previous sections) in order to estimate the absolute values of the tissue oxygenation status (tissue saturation index or tissue oxygenation index).

Regarding detectors, various semiconductor photodiodes are available, usually offering very good dynamic range at low cost. $^{27,29,30,35}$ Avalanche photodiodes ${ }^{32,34}$ are generally the most sensitive among the semiconductor detectors. However, for larger sensitivity areas, photomultipliers (PMTs) ${ }^{33}$ are required.

CW systems can provide very fast sampling rates: up to $100 \mathrm{~Hz}$ when few channels are used down to $10 \mathrm{~Hz}$ when imaging system are employed.

\section{Frequency domain instruments}

In vivo FD measurements were introduced in the 1990s (see for example Lakowicz and Berndt ${ }^{45}$ and Chance et al. ${ }^{46}$ ). FD instruments were used for both muscle and brain studies (for examples see reviews on NIR spectroscopy ${ }^{39-45}$ and more recent applications of this kind of instrumentation ${ }^{47-59}$ ). An example of an FD instrument is also commercially available. ${ }^{61}$

In FD regime, sources are modulated at a few hundreds of megahertz. It is possible to modulate a CW source using an external device based on the electro-optical or the acoustooptical effect. ${ }^{60}$ It is easier and more cost effective to directly modulate the sources; for example, driving diode lasers with amplitude modulated current, reaching a bandwidth of gigahertz, or light-emitting diodes, reaching a bandwidth of hundreds of megahertz. However, both for external and internal modulation, systems require a radio-frequency oscillator to drive the source and to provide a reference signal for the phase measurement. The wavelengths used are the same as those employed in CW instruments. The most common commercial systems ${ }^{61}$ work with two wavelengths $(690 \mathrm{~nm}$ and $830 \mathrm{~nm})$.

In principle, as for CW systems, in the FD it is possible to encode wavelengths and positions using a proper modulation frequency in the $\mathrm{MHz}$ rang $\mathrm{e}^{62}$ but, in $\mathrm{FD}$, the complexity of the acquisition electronics increases.

All the detectors used for CW measurements can also be employed for FD measurements. The faster the detector, the higher is the achievable modulation bandwidth. ${ }^{60}$ For example, solid state detectors (photodiodes, avalanche photodiodes) are faster than PMTs, allowing bandwidths of up to the gigahertz range. In FD, detectors are part of a demodulation electronic chain. There are two different approaches: internal and external demodulation. In the former scheme, detector gain is directly modulated at the proper frequency; in this way, the electrical output of the detector is naturally demodulated. In the latter scheme, the gain of the detector is constant and its output is demodulated using an external electronic mixer. Heterodyning is commonly performed to convert the radiofrequency to a few kilohertz prior to phase detection. The detected signal is digitised over an appropriate period of time, and phase and amplitude are computed. ${ }^{10}$

FD systems are inexpensive, stable and can provide fast sampling (up to $50 \mathrm{~Hz}$ ). Thus FD systems are well suited to acquiring measurements quickly and at relatively highdetected intensities. FD systems can be easily miniaturised and take advantage of highly optimised components. Further, the low-frequency noise is naturally suppressed because of the signal modulation and the subsequent demodulation, reaching a very high signal-to-noise ratio if compared to $\mathrm{CW}$ or TD systems. On the other hand, when the signal is very low, it can only be detected using the powerful pulsed laser sources and photon counting techniques incorporated into TD systems. A variety of frequency domain systems have been developed for both optical topography ${ }^{63,64}$ and optical tomography. ${ }^{65}$

\section{Time domain instruments}

In vivo TD measurements were introduced at the end of the 1980s (see for example Chance et al. ${ }^{66}$ and Delpy et al. ${ }^{67}$ ). In the literature, in comparison to the high number of studies employing CW and FD instruments, there are few papers regarding TD functional NIR spectroscopy for brain and muscle studies. ${ }^{68-79}$ Furthermore, to our knowledge, only one commercial system, ${ }^{80}$ available only in Japan, (TRS-20, Hamamatsu Ltd, Japan) works in the TD regime.

In TD NIR measurements, the instrument itself distorts the TPSF, because its pulse response is not a delta function, but shows a certain shape. To take this aspect into account, the theoretical model must be convolved with the instrument response function. ${ }^{81}$ The narrower the instrument response function, the better is the accuracy of FIT, an important consideration during the design of the instrument. The 
instrument response function shape depends on three main components of the instrumentation: light source lfor example, diode lasers show larger pulses than other kinds of laser and are more expensive and bulky), detectors (for example, solid state detectors show a narrower pulse response than photomultipliers, but smaller active areal and light delivery fibres (fibres with lower numerical aperture have less modal dispersion but also lower light collection efficiencyl. Each of these three parts can be tailored in order to optimise the instrument response function.

For this reason, in TD systems the source is a pulsed laser with high repetition frequency (>40 MHz), narrow full width at half maximum (<150 ps), sufficient output average power labout $1 \mathrm{~mW}$ ) and small dimensions to be used in a clinical environment. Different groups have built TD systems employing semiconductor pulsed lasers ${ }^{80,82-85}$ which are very compact and robust for clinical use, but are associated with some drawbacks in terms of available average power with narrow pulses. As for CW instruments, in the TD we observe two different approaches in the choice of wavelengths. In fact, in some instruments, only two wavelength ${ }^{82}$ are employed in order to reduce the complexity of the system, while in some other cases, the employed wavelengths are three 80,83 to strengthen the accuracy of the haemoglobin concentration estimation. Independently of the number of laser sources, there are two possible injection strategies: time multiplexing or space multiplexing. ${ }^{86}$ In the former, all the wavelengths are injected in the same point but temporally delayed (some nanoseconds) and the detected photons are discriminated by means of their arrival time. The only constraint is that the delay between pulses at different wavelengths is greater than the broadening experienced by the pulse travelling into the tissue but lower than the repetition period of the pulsed source; hence the overlap between different wavelengths or between different pulse repetitions is avoided. Conversely, in the second strategy, the different wavelengths are injected in different points at the same time and, when a sufficient amount of signal is recorded, the wavelengths are exchanged; at the end of the measurement for each spatial point there are TPSFs at all the wavelengths. The only constraint is that the signal-to-noise ratio must be sufficient to collect all the TPSFs in $1 \mathrm{~s}$ or less in order to be sure that the haemodynamic status of the sample does not change during the measurement, otherwise the TPSFs at the different wavelengths cannot be considered simultaneous in the haemodynamic time scale. ${ }^{86}$

To measure the TPSF, a detector and an acquisition system with a temporal resolution of picoseconds is necessary. The first detector with these characteristics employed in a TD system was the Streak Camera that achieves a temporal resolution of $1-10 \mathrm{ps} .{ }^{87}$ The principal disadvantages of streak cameras are the high cost and relatively small effective light collection area. ${ }^{88}$ Despite this, as a result of the unrivaled temporal resolution and to the ability to easily perform broadband multi-wavelength measurements, streak camera-based set-ups are currently being used for characterisation of diffu- sive media. ${ }^{89-91}$ However, high costs limit the use of streak cameras for clinical applications.

An alternative means of measuring entire TPSFs is a device based on the TCSPC technique. The TCSPC technique exploits the fact that, for low level and high-repetition-rate signals, the light intensity is so low that the probability of detecting one photon in one signal period is far less than 1. Thus, it is sufficient to record the photons, measure their arrival time in the signal period (i.e. the time interval between the injection of two laser pulses into the sample) and store this information. After the detection of many photons, the distribution of the detection times (i.e. the waveform of the optical pulse) builds up. A pulsed laser source with a repetition rate of tens of megahertz is required in order to reduce the recording time of the entire TPSF. The arrival time of the individual single photon should be measured with high precision. The bandwidth of a photon counting experiment is therefore limited only by the transit time spread of the detectors and not by the width of the single-photon pulses (the single electron response). The transit time spread is usually one order of magnitude narrower than the single electron response; thus TCSPC obtains a significantly higher time-resolution than any analogue recording technique. This technique generally requires a photomultiplier tube as detector. A PMT can be broken by an excess of light; for this reason, systems employing these detectors need to be protected to avoid excessive light exposure. PMTs for single photon counting with transit time spread of 150-200 ps are available, but for a significantly shorter transit time spread ( $<50$ ps) it is necessary to employ a microchannel-plate PMT. Compared to Streak Camera, TCSPC systems offer a superior light collection area at a substantially reduced cost. However, only in the last decade, TCSPC has replaced the Streak Camera because the maximum count rate of the recording electronics has increased by two orders of magnitude. State-of-the-art TCSPC devices work at count rates of several million photons per second. Since, in good instruments, the noise is mainly due to shot noise, this means that time domain instrumentation has an inherently low signal-to-noise ratio compared to other systems that can handle orders of magnitudes more photons (such as FD and CW systems). For a more detailed description of the TCSPC technique see Becker. ${ }^{92}$

TD systems can also perform imaging by multiplying the number of measurement points. This can be obtained by increasing the number of injection points and activating them sequentially; for example, by means of an optical switch ${ }^{82,83,85}$ and by increasing the number of independent simultaneously operating detectors ${ }^{82,84}$ that can operate simultaneously. This approach is limited by the achievable acquisition rate and the instrument dimensions. In fact, by multiplying the number of injection points, the total amount of measuring time increases because all the TPSF acquisitions are performed sequentially. While increasing the number of independent detectors, the measuring time does not change, but the instrument becomes bulky. Different solutions have been implemented in the construction of TD imaging systems: increasing the number of injection points but maintaining a low number of 
detection channels ${ }^{83}$ or increasing both detectors and injection points ${ }^{82}$ reaching the limit in terms of tolerable dimensions and complexity of the system. Whatever the adopted scheme, the achievable acquisition rate, with a good signalto-noise ratio, with this typology of instrument, is around $1 \mathrm{~Hz}$ with the maximum number of measurement points (tens of points). By reducing the number of points, the acquisition rate can be reduced down to 50-100 ms. Differently from CW and FD, where modulation is used to encode wavelengths and place, in TD it is impossible to collect photons in the same detectors coming from more than one injection point at the same time because it would be impossible to distinguish between photons coming from different locations. Recently, a novel approach to TD measurements was proposed in which the pulsed source is modulated with a pseudo random code obtaining the equivalent in TD of the light modulation in the CW or FD regime, thus allowing the simultaneous acquisition at each detection point of the photons coming from all the injection points. ${ }^{93}$ This type of detection scheme needs further testing before being implemented in a clinical instrument.

As observed in the previous theoretical paragraphs, the depth sensitivity of the TD approach does not depend on the distance ${ }^{94}$ between injection and detection points; by exploiting this feature, a novel class of prototypes was born. ${ }^{95-97}$ The key advantages of these systems are: the increase of spatial resolution and achievable signal-to-noise ratio thanks to the small distance between injection an detection points. Up to now this novel type of TD instrument is at a prototype level and no clinical versions are available. However, the possibility to perform a TD measurement placing injection and detection fibres one close to the other is promising.

\section{Conclusions}

We have reviewed theories and their instrumental implementations used for studying the oxygenation of muscle and brain cortex. In particular, we considered three different approaches for functional near infrared spectroscopy: the CW technique, FD technique and TD technique. We have analysed the common layout of these instruments, focusing our attention on their main components: sources, injection and collection optics, detection and acquisition electronics. Starting from a theoretical standpoint, we have highlighted the different technical choices implemented in these three types of spectroscopy showing their main features, similarities and drawbacks.

\section{Acknowledgements}

We wish to acknowledge partial support from the European Community's Seventh Framework Programme (FP7/20072013) under grant agreement $n^{\circ}$ FP7-HEALTH-F5-2008-201076.

\section{References}

1. C.E. Elwell and C.E. Cooper, "Making light work: Illuminating the future of biomedical optics", Philos. Trans. R. Soc. Lond. A 369, 4358 (2011). doi: 10.1098/ rsta.2011.0302

2. F. Martelli, S. Del Bianco, A. Ismaelli and G. Zaccanti, Light Propagation through Biological Tissue and Other Diffusive Media: Theory, Solutions and Software. SPIE Press, Bellingham, WA, USA (2010). doi: $10.1117 / 3.824746$

3. F. Martelli, "ABC of near infrared photon migration in tissues: the diffusive regime of propagation", J. Near Infrared Spectrosc. 20(1), XX (2012). doi:

4. B.L. Horecker, "The absorption spectra of hemoglobin and its derivatives in the visible and near infra-red regions", J. Biol. Chem. 148, 173 (1943).

5. M. Cope, D.T. Delpy, S. Wray, J.S. Wyatt and E.O. Reynolds, “A CCD spectrophotometer to quantitate the concentration of chromophores in living tissue utilising the absorption peak of water at $975 \mathrm{~nm}$ ", Adv. Exp. Med. Biol. 248, 33 (1989).

6. E.O.R. Reynolds, J.S. Wyatt, D. Azzopardi, D.T. Delpy, E.B. Cady, M. Cope and S. Wray, "New non-invasive methods for assessing brain oxygenation and hemodynamics", Br. Med. Bull. 44, 1052 (1988).

7. H. Obrig and A. Villringer, "Beyond the visibleimaging the human brain with light", J. Cereb. Blood Flow Metab. 23, 1 (2003). doi: 10.1097/00004647200301000-00001

8. M.S. Patterson, E. Schwartz and B.C. Wilson, "Quantitative reflectance spectrophotometry for the noninvasive measurement of photosensitizer concentration in tissue during photodynamic therapy", Proc. SPIE 1065, 115 (1989).

9. S.J. Matcher, M. Cope and D.T. Delpy, "Quantification of tissue chromophore concentration via water-peak measurements in near-infrared spectroscopy", Proc. SPIE 1888, 239 (1993).

10. A.P. Gibson, J.C. Hebden and S.R. Arridge, "Recent advances in diffuse optical imaging", Phys. Med. Biol. 50, R1 (2005). doi: 10.1088/0031-9155/50/4/R01

11. S.R. Arridge, "Photon-measurement density-functions. 1. Analytical forms", Appl. Opt. 34, 7395 (1995). doi: 10.1364/A0.34.007395

12. S.R. Arridge and M. Schweiger, "Photon-measurement density-functions. 2. Finite-element-method calculations", Appl. Opt. 34, 8026 (1995). doi: $\underline{10.1364 /}$ A0.34.008026

13. S.R. Arridge and W.R.B. Lionheart, "Nonuniqueness in diffusion-based optical tomography", Opt. Lett. 23, 882 (1998). doi: 10.1364/OL.23.000882

14. S.L. Jacques, "Time resolved propagation of ultrashort laser-pulses within turbid tissues", Appl. Opt. 28, 2223 (1989). doi: $10.1364 / \mathrm{AO} .28 .002223$

15. M.S. Patterson, B. Chance and B.C. Wilson, "Time resolved reflectance and transmittance for the 
noninvasive measurement of tissue optical-properties", Appl. Opt. 28, 2331 (1989). doi: 10.1364/A0.28.002331

16. D. Comelli, A. Bassi, A. Pifferi, P. Taroni, A. Torricelli, R. Cubeddu, F. Martelli and G. Zaccanti, "In vivo timeresolved reflectance spectroscopy of the human forehead", Appl. Opt. 46, 1717 (2007). doi: 10.1364 / A0.46.001717

17. Q. Fang and D.A. Boas, "Monte Carlo simulation of photon migration in 3D turbid media accelerated by graphics processing units", Opt. Exp. 17, 20178 (2009). doi: 10.1364/OE.17.020178

18. D. Contini, L. Spinelli, A. Torricelli, A. Pifferi and R. Cubeddu, "Novel method for depth-resolved brain functional imaging by time-domain NIRS", Proceedings of SPIE-OSA, Biomed. Opt. Opt. Soc. Am. 6629, 7 (2007).

19. J. Steinbrink, H. Wabnitz, H. Obrig, A. Villringer and H. Rinneberg, "Determining changes in NIR absorption using a layered model of the human head", Phys. Med. Biol. 46, 879 (2001). doi: 10.1088/0031-9155/46/3/320

20. A. Liebert, H. Wabnitz, J. Steinbrink, H. Obrig, M. Moller, R. Macdonald, A. Villringer and H. Rinneberg, "Timeresolved multidistance near-infrared spectroscopy of the adult head: intracerebral and extracerebral absorption changes from moments of distribution of times of flight of photons", Appl. Opt. 43, 3037 (2004). doi: 10.1364/ A0.43.003037

21. M.S. Patterson, J.D. Moulton, B.C. Wilson, K.W. Berndt and J.R. Lakowicz, "Frequency-domain reflectance for the determination of the scattering and absorption properties of tissue", Appl. Opt. 30, 4474 (1991). doi: $10.1364 /$ A0.30.004474

22. Y. Yamashita, A. Maky and H. Koizumi, "Wavelength dependence of the precision of noninvasive optical measurement of oxy-, deoxy- and total-hemoglobin concentration", Med. Phys. 28, 1108 (2001). doi: 10.1118/1.1373401

23. G. Strangman, M.A. Franceschini and D.A. Boas, "Factors affecting the accuracy of near-infrared spectroscopy calculations for focal changes in oxygenation parameters", Neuroimage 18, 865 (2003). doi: 10.1016/ S1053-8119(03)00021-1

24. D.A. Boas, A.M. Dale and M.A. Franceschini, "Diffuse optical imaging of brain activation: approaches to optimizing image sensitivity, resolution and accuracy", Neuroimage 23, 275 (2004). doi: 10.1016/j.neuroimage.2004.07.011

25. M. Ferrari and V. Quaresima, "Near-infrared brain and muscle oximetry: from the discovery to current applications", J. Near Infrared Spectrosc. 20(1), XX (2012). doi:

26. S. Cutini, S. Basso Moro and S. Bisconti, "Functional near-infrared optical imaging in cognitive neuroscience: An introductory review", J. Near Infrared Spectrosc. 20(1), XX (2012). doi: $\underline{10.1255 / \text { jnirs.969 }}$

27. Portamon (www.artinis.com).

28. fNIR 100 and fNIR 200 (www.biopac.com).

29. NIRScout (www.nirx.net).
30. Hb-12c (www.astem-jp.com).

31. Equanox (www.noninequanox.com).

32. Oxymon Mk III (www.artinis.com)

33. Foire 3000 (www.shimadzu.com).

34. ETG 4000 (www.hitachi-medical-systems.eu).

35. NIRO 200 (www.jp.hamamatsu.com).

36. NIMO (www.nimoworld.com).

37. FORE-SIGHT (www.casmed.com).

38. M. Wolf, M. Ferrari and V. Quaresima, "Progress of near-infrared spectroscopy and topography for brain and muscle clinical applications", J. Biomed. Opt. 12(6), 062104 (2007). doi: 10.1117/1.2804899

39. M. Calderon-Arnulphi, A. Alaraj and K.V. Slavin, "Near infrared technology in neuroscience: Past, present and future", Neurol. Res. 31(6), 605 (2009). doi: 10.1179/174313209X383286

40. D.T. Delpy and M. Cope, "Quantification in tissue near infrared spectroscopy", Philos. Trans. R. Soc. Lond. B., Biol. Sci. 352(1354), 649 (1997). doi: $10.1098 /$ rstb.1997.0046

41. M. Ferrari, L. Mottola and V. Quaresima, "Principles, techniques and limitations of near infrared spectroscopy", Can. J. Appl. Physiol. 29(4), 463 (2004). doi: 10.1139/h04-031

42. A. Pellicer and M. del C. Bravo, "Near-infrared spectroscopy: A methodology-focused review", Semin. Fetal Neonatal Med. 16(1), 42 (2011). doi: 10.1016/j. siny.2010.05.003

43. T. Hamaoka, K.K. McCully, V. Quaresima, K. Yamamoto and B. Chance, "Near-infrared spectroscopy/imaging for monitoring muscle oxygenation and oxidative metabolism in healthy and diseased humans", J. Biomed. Opt. 12(6), 062105 (2007). doi: 10.1117/1.2805437

44. M. Ferrari, M. Muthalib and V. Quaresima, "The use of near-infrared spectroscopy in understanding skeletal muscle physiology: recent developments", Philos. Trans. R. Soc. Lond. A 369, 4577 (2011).

45. J.R. Lakowicz and K. Berndt, "Frequency domain measurement of photon migration in tissue", Chem. Phys. Lett. 166, 246 (1990). doi: 10.1016/0009-2614(90)80024-8

46. B. Chance, M. Cope, E. Gratton, N. Ramanujam and B. Tromberg, "Phase measurement of light absorption and scatter in human tissue", Rev. Sci. Instr. 69, 3457 (1998). doi: $\underline{10.1063 / 1.1149123}$

47. B.J. Lutjemeier, L.F. Ferreira, D.C. Poole, D. Townsend and T.J. Barstow, "Muscle microvascular hemoglobin concentration and oxygenation within the contractionrelaxation cycle", Respir. Physiol. Neurobiol. 160, 131 (2008). doi: 10.1016/j.resp.2007.09.005

48. L.F. Ferreira, D.M. Hueber and T.J. Barstow, "Effect of assuming constant optical scattering on measurements of muscle oxygenation by near-infrared spectroscopy during exercise", J. Appl. Physiol. 102, 358 (2007). doi: 10.1152/japplphysiol.00920.2005

49. Y. Tong, P.R. Bergethon and B.B. Frederick, “An improved method for mapping cerebrovascular reserve 
using concurrent fMRI and near-infrared spectroscopy with regressor interpolation at progressive time delays (RIPTiDe)", Neuroimage 56, 2047 (2011). doi: 10.1016/j. neuroimage.2011.03.071

50. U. Chaudhary, M. Hall, J. DeCerce, G. Rey and A. Godavarty, "Frontal activation and connectivity using near-infrared spectroscopy: Verbal fluency language", Brain Res. Bull. 84, 197 (2011). doi: 10.1016/j.brainresbull.2011.01.002

51. S. Lee, M. Lee, D. Koh, B.M. Kim and J.H. Choi, "Cerebral hemodynamic responses to seizure in the mouse brain: simultaneous near-infrared spectroscopyelectroencephalography study", J. Biomed. Opt. 15, 037010 (2010). doi: $\underline{\text { 10.1117/1.3365952 }}$

52. C. Im, Y. Jung, S. Lee, D. Koh, D. Kim and B. Kim, "Estimation of directional coupling between cortical areas using near-infrared spectroscopy (NIRS)", Opt. Expr. 18, 5730 (2010). doi: 10.1364/OE.18.005730

53. K. Kotilahti, I. Nissila, T. Nasi, L. Lipiainen, T. Noponen, P. Merilainen, M. Huotilainen and V. Fellman, "Hemodynamic responses to speech and music in newborn infants", Hum. Brain Mapp. 31, 595 (2010).

54. Y. Tong and B.B. Frederick, "Time lag dependent multimodal processing of concurrent fMRI and near-infrared spectroscopy (NIRS) data suggests a global circulatory origin for low-frequency oscillation signals in human brain", Neuroimage 53, 553 (2010). doi: 10.1016/j.neuroimage.2010.06.049

55. B. Zhu, N. Yadav, G. Rey and A. Godavarty, "Diffuse optical imaging of brain activation to joint attention experience", Behav. Brain Res. 202, 32 (2009). doi: 10.1016/j.bbr.2009.03.029

56. N. Roche-Labarbe, S.A. Carp, A. Surova, M. Patel, D.A. Boas, P.E. Grant and M.A. Franceschini, "Noninvasive optical measures of $\mathrm{CBV}, \mathrm{StO}_{2}, \mathrm{CBF}$ index and $\mathrm{rCMRO} 2$ in human premature neonates' brains in the first six weeks of life", Hum. Brain Mapp. 31, 341 (2010). doi: $10.1002 / \mathrm{hbm} .20868$

57. H. Radhakrishnan, W. Vanduffel, H.P. Deng, L. Ekstrom, D.A. Boas and M.A. Franceschini, "Fast optical signal not detected in awake behaving monkeys", Neuroimage 45, 410 (2009). doi: 10.1016/j. neuroimage.2008.12.014

58. A. Gallagher, M. Lassonde, D. Bastien, P. Vannasing, F. Lesage, C. Grova, A. Bouthillier, L. Carmant, F. Lepore, R. Béland and D.K. Nguyen, “Non-invasive pre-surgical investigation of a 10-year-old epileptic boy using simultaneous EEG-NIRS", Seizure 17, 576 (2008). doi: 10.1016/j. seizure.2008.01.009

59. N. Jausovec and K. Jausovec, "Gender related differences in visual and auditory processing of verbal and figural tasks", Brain Res. 1300, 135 (2009).

60. S. Fantini and M.A. Franceschini, "Frequency-domain techniques for tissue spectroscopy and imaging", in
Handbook of Optical Biomedical Diagnostics, Ed by V.V. Tuchin. SPIE Press, Bellingham, WA, USA, p. 405 (2002).

61. Oxiplex TS and Imagent (www.iss.com).

62. G. Yu, T. Durduran, D. Furuya, J.H. Greenberg and A.G Yodh, "Frequency-domain multiplexing system for in vivo diffuse light measurements of rapid cerebral hemodynamics", Appl. Opt. 42, 2931 (2003). doi: $10.1364 /$ $\underline{\text { A } 0.42 .002931}$

63. R.M. Danen, Y. Wang, X.D. Li, W.S. Thayer and A.G. Yodh, "Regional imager for low-resolution functional imaging of the brain with diffusing near-infrared light", Photochem. Photobiol. 67, 33 (1998). doi: 10.1111/j.1751-1097.1998. tb05162.x

64. M.A. Franceschini, V. Toronov, M.E. Filiaci, E. Gratton and S. Fantini, "On-line optical imaging of the human brain with 160-ms temporal resolution", Opt. Expr. 6, 49 (2000). doi: $10.1364 / 0 E .6 .000049$

65. B.W. Pogue, S. Geimer, T.O. McBride, S.D. Jiang, U.L. Osterberg and K.D. Paulsen, "Three-dimensional simulation of near-infrared diffusion in tissue: Boundary condition and geometry analysis for finite-element image reconstruction", Appl. Opt. 40, 588 (2001). doi: 10.1364/A0.40.000588

66. B. Chance, J.S. Leigh, H. Miyake, D.S. Smith, S. Nioka, R. Greenfeld, M. Finander, K. Kaufmann, W. Levy, M. Young, P. Choen, H. Yoshioka and R. Boretsky, "Comparison of time resolved and unresolved measurements of deoxyhaemoglobin in brain", Proc. Natl. Acad. Sci. USA 85, 4971 (1988). doi: 10.1073/pnas.85.14.4971

67. D.T. Delpy, M. Cope, P. van der Zee, S. Arridge, S. Wray and J. Wyatt, "Estimation of optical path-length through tissue from direct time-of-flight measurement", Phys. Med. Biol. 33, 1433 (1988). doi: 10.1088/00319155/33/12/008

68. S. Ferrante, D. Contini, L. Spinelli, A. Pedrocchi, A. Torricelli, F. Molteni, G. Ferrigno and R. Cubeddu, "Monitoring muscle metabolic indexes by time-domain near-infrared spectroscopy during knee flex-extension induced by functional electrical stimulation", J. Biomed. Opt. 14, 044011 (2009). doi: 10.1117/1.3183802

69. M. Belau, M. Ninck, G. Hering, L. Spinelli, D. Contini, A. Torricelli and T. Gisler, "Noninvasive observation of skeletal muscle contraction using near-infrared timeresolved reflectance and diffusing-wave spectroscopy", J. Biomed. Opt. 15, 057007 (2010). doi: 10.1117/1.3503398

70. E. Yamada, T. Kusaka, N. Arima, K. Isobe, T. Yamamoto and S. Itoh, "Relationship between muscle oxygenation and electromyography activity during sustained isometric contraction", Clin. Physiol. Funct. Imag. 28, 216 (2008). doi: 10.1111/j.1475-097X.2008.00798.x

71. T.H. Sander, S. Leistner, H. Wabnitz, B. MacKert, R. MacDonald and L. Trahms, "Cross-correlation of motor activity signals from dc-magnetoencephalography, nearinfrared spectroscopy and electromyography", Comput. Intell. Neurosci. 2010, 10 (2010). doi: 10.1155/2010/785279 
72. N. Yokose, K. Sakatani, Y. Murata, T. Awano, T. Igarashi S. Nakamura, T. Hoshino and Y. Katayama, "Bedside monitoring of cerebral blood oxygenation and hemodynamics after aneurysmal subarachnoid hemorrhage by quantitative time-resolved near-infrared spectroscopy", World Neurosurg. 73, 508 (2010). doi: 10.1016/j. wneu.2010.02.061

73. M. Butti, D. Contini, E. Molteni, M. Caffini, L. Spinelli, G. Baselli, A.M. Bianchi, S. Cerutti, R. Cubeddu and A. Torricelli, "Effect of prolonged stimulation on cerebral hemodynamic: A time-resolved fNIRS study", Med. Phys. 36, 4103 (2009). doi: 10.1118/1.3190557

74. M. Diop, K.M. Tichauer, J.T. Elliott, M. Migueis, T. Lee and K.S. Lawrence, "Comparison of time-resolved and continuous-wave near-infrared techniques for measuring cerebral blood flow in piglets", J. Biomed. Opt. 15, 057004 (2010). doi: 10.1117/1.3488626

75. L. Gagnon, C. Gauthier, R.D. Hoge, F. Lesage, J. Selb and D.A. Boas, "Double-layer estimation of intra- and extracerebral hemoglobin concentration with a timeresolved system", J. Biomed. Opt. 13, 054019 (2008). doi: 10.1117/1.2982524

76. B. Mackert, S. Leistner, T. Sander, A. Liebert, H. Wabnitz, M. Burghoff, L. Trahms, R. Macdonald and G. Curio, “Dynamics of cortical neurovascular coupling analyzed by simultaneous DC-magnetoencephalography and time-resolved near-infrared spectroscopy", Neuroimage 39, 979 (2008). doi: 10.1016/j.neuroimage.2007.09.037

77. T. Austin, J.C. Hebden, A.P. Gibson, G. Branco, R. Yusof, S.R. Arridge, J.H. Meek, D.T. Delpy and J.S. Wyatt,

"Three-dimensional optical imaging of blood volume and oxygenation in the preterm brain", Neuroimage 31, 1426 (2006). doi: 10.1016/j.neuroimage.2006.02.038

78. A. Torricelli, V. Quaresima, A. Pifferi, G. Biscotti, L. Spinelli, P. Taroni, M. Ferrari and R. Cubeddu, "Mapping of calf muscle oxygenation and haemoglobin content during dynamic plantar flexion exercise by multichannel time-resolved near infrared spectroscopy", Phys. Med. Biol. 49, 685 (2004). doi: 10.1088/00319155/49/5/003

79. V. Quaresima, M. Ferrari, A. Torricelli, L. Spinelli, A. Pifferi and R. Cubeddu, "Bilateral prefrontal cortex oxygenation responses to a verbal fluency task: a multichannel time-resolved near-infrared topography study", J. Biomed. Opt. 10(1), 11012 (2005). doi: 10.1117/1.1851512

80. TRS-20 (www.hamamatsu.com).

81. A. Torricelli, D. Contini, L. Spinelli, M. Caffini, A. Pifferi and R. Cubeddu, "Advanced optical methods for functional brain imaging: Time domain functional near infrared spectroscopy", in Advances in Optical Imaging for Clinical Medicine, Ed by N. Iftimia, W.R. Brugge and D.X. Hammer. John Wiley \& Sons Inc., Hoboken, USA, p. 287 (2011).

82. D. Contini, A. Torricelli, A. Pifferi, L. Spinelli, F. Paglia and R. Cubeddu, "Multi-channel time-resolved system for functional near infrared spectroscopy”, Opt. Exp. 14, 5418 (2006). doi: 10.1364/OE.14.005418
83. H. Wabnitz, M. Moeller, A. Liebert, A. Walter, R. Erdmann, O. Raitza, C. Drenckhan, J.P. Dreier, H. Obrig, J. Steinbrink and R. MacDonald, “A time-domain NIR brain imager applied in functional stimulation experiments", Proc. SPIE 5859 (2005).

84. K. Wells, J.C. Hebden, F.E.W. Schmidt and D.T. Delpy, "The UCL multichannel time-resolved system for optical tomography", Proc. SPIE 2979, 599 (1997). doi: 10.1117/12.280298

85. M. Kacprzak, A. Liebert, P. Sawosz, N. Zolek and R. Maniewski, "Time-resolved optical imager for assessment of cerebral oxygenation", J. Biomed Opt. 12, 034019 (2007). doi: $\underline{10.1117 / 1.2743964}$

86. R. Re, D. Contini, M. Caffini, R. Cubeddu, L. Spinelli and A. Torricelli, "A compact time-resolved system for near infrared spectroscopy based on wavelength space multiplexing", Rev. Sci. Instrum. 81, 113101 (2010). doi: $10.1063 / 1.3495957$

87. D.T. Delpy, M. Cope, P. Van der Zee, S. Arridge, S. Wray and J. Wyatt, "Estimation of optical path-length through tissue from direct time of flight measurement" Phys. Med. Biol. 33, 1433 (1988). doi: 10.1088/0031-9155/33/12/008

88. J.C. Hebden, S.R. Arridge and D.T. Delpy, "Optical imaging in medicine: I. Experimental techniques", Phys. Med. Biol. 42, 825 (1997).

doi: $10.1088 / 0031-9155 / 42 / 5 / 007$

89. C. Abrahamsson, T. Svensson, S. Svanberg and S. Andersson-Engels, "Time and wavelength resolved spectroscopy of turbid media using light continuum generated in a crystal fiber" Opt. Exp. 12, 4103 (2004). doi: 10.1364/OPEX.12.004103

90. C.V. Zint, W. Uhring, M. Torregrossa, B. Cunin and P. Poulet, "Streak camera: A multidetector for diffuse optical tomography", Appl. Opt. 42, 3313 (2003). doi: $10.1364 /$ A0.42.003313

91. R. Esposito, S. De Nicola, M. Lepore, I. Delfino and P. L. Indovina, "A perturbation approach to characterize absorptive inclusions in media by time-resolved contrast measurements", J. Opt. A Pure Appl. Opt. 6, 736 (2004). doi: $10.1088 / 1464-4258 / 6 / 7 / 013$

92. W. Becker, Advanced Time-Correlated Single Photon Counting Techniques. Springer Verlag, Berlin, Germany (2005). doi: $10.1007 / 3-540-28882-1$

93. N.G. Chen and Q. Zhu, "Time-resolved diffusive optical imaging using pseudo-random bit sequences", Opt. Exp. 11, 3445 (2003). doi: 10.1364/OE.11.003445

94. A. Torricelli, A. Pifferi, L. Spinelli, R. Cubeddu, F. Martelli, S. Del Bianco and G. Zaccanti, "Time-resolved reflectance at null source-detector separation: improving contrast and resolution in diffuse optical imaging", Phys. Rev. Lett. 95, 078101 (2005). doi: 10.1103/ PhysRevLett.95.078101

95. A. Pifferi, A. Torricelli, L. Spinelli, D. Contini, R. Cubeddu, F. Martelli, G. Zaccanti, A. Tosi, A. Dalla Mora, F. Zappa and S. Cova, "Time-resolved diffuse reflectance using small source detector separation and fast single-photon 
gating", Phys. Rev. Lett. 100, 138101 (2008). doi: $10.1103 /$ PhysRevLett.100.138101

96. A. Dalla Mora, A. Tosi, F. Zappa, S. Cova, D. Contini, A. Pifferi, L. Spinelli, A. Torricelli and R. Cubeddu, "Fastgated single photon avalanche diode for wide dynamic range near infrared spectroscopy" IEEE JSTQE 16, 1023 (2010).
97. A. Tosi, A. Dalla Mora, F. Zappa, A. Gulinatti, D. Contini, A. Pifferi, L. Spinelli, A. Torricelli and R. Cubeddu, "Fastgated single photon counting technique widens dynamic range and speeds up acquisition time in time-resolved measurements", Opt. Exp. 19, 10735 (2011). doi: $10.1364 /$ $\underline{\text { OE.19.010735 }}$ 
